\title{
La creación de valor en el museo y la sociomuseología
}

\section{The Creation of Value in the Museum and Sociomuseology}

\author{
Ana Mercedes Fernández Dos Reis Díez \\ Universidade Lusófona de Lisboa \\ Rua das Musas 3.04.01C $1^{\circ}$ Esq. \\ 1990 - 169 Lisboa, Portugal \\ mercedes.stoffel@gmail.com
}

Recibido: 24-04-2014

Aceptado: 30-06-2014

\begin{abstract}
RESUMEN
Los museos tienen normalmente mucha dificultad en justificar su existencia y en demostrar su valor real y su contribución al desarrollo de las comunidades a las que sirven. El coste del mantenimiento de un museo, después de inaugurado, nunca parece estar justificado para las tutelas y financiadores que lo promovieron.

El museo, grande o pequeño, puede ser un elemento esencial en el desarrollo cultural del conjunto social, pero necesita tener una actividad que lo justifique y que su labor sea reconocida. Para contribuir con eficacia debe compartir con la comunidad su misión y sus actividades y para probar su valor debe tener sistemas de evaluación que permitan verificar si su labor de investigación y acción sobre el patrimonio, de promoción de la cultura y la ciudadanía y de colaboración con escuelas y asociaciones, reduce el fracaso escolar, aumenta la participación en la vida y cultura o contribuye a la cohesión social y a la formación de ciudadanos más responsables y solidarios. La Sociomuseología estudia estos campos de la actividad del museo, acompaña experiencias y propone soluciones a estas dificultades.
\end{abstract}

Palabras Clave: Sociomuseología. Valor Compartido. Evaluación. Desarrollo.

\begin{abstract}
Museums normally have difficulty in accounting for their existence and in demonstrating cost of keeping a museum open after its inauguration seems it is never justifiable for the political powers and the financing entities that were their first developers.

The museum, big or small, can be an essential element in the development of society as a whole but it needs to transform its activity and direct it to this goal and needs its work to be publicly recognized. In order to contribute with specific results to society, the museum must share its mission and activities with the community and to prove its value it must have quality systems that are able to evaluate if its investigation and action over cultural heritage, its promotion of culture and citizenship and its collaboration with schools and associations reduces school failure, increases participation in social and cultural life or contributes to the cohesion of society and to the education of more responsible and supportive citizens. Social museology science studies these fields of activity of museums, it accompanies experiences and proposes solutions.
\end{abstract}

Key words: Social Museology. Shared Vale. Evaluation. Development. 


\section{Museología y desempeño social de los mu- seos}

De una forma simplificada, podríamos decir que los museos son centros de preservación, investigación y divulgación del patrimonio de todos. Como las escuelas o las bibliotecas, tienen el encargo de producir cultura para los ciudadanos y utilizar recursos para ese fin. Pero si rara vez se anatematiza una escuela o una biblioteca como no rentable, y su funcionamiento se acepta y se sufraga pacíficamente, en lo que se refiere a los museos, esta aceptación no está de ninguna manera garantizada. Y si parece fácil reconocer el valor y la contribución al desarrollo y el bien común de las bibliotecas y las escuelas, no existe la misma percepción, por parte del estado y de la comunidad en general, cuando se trata de evaluar los méritos de un museo. Parece, por tanto, en una lógica puramente financiera, que apenas los grandes museos, detentores de importantes bienes histórico/artísticos o científicos, que poseen patrimonios espectaculares y poderosos despliegues de medios para el turismo de masas, serán capaces de generar ingresos directos e indirectos justificados para sus promotores $y$, en consecuencia, de sobrevivir.

Los museos nacieron en el siglo de las luces, como sabemos, para divulgar la cultura dominante de entonces entre las poblaciones recién llegadas a la ciudadanía. Sin embargo, muy pronto, los responsables de la conservación de aquellos bienes artísticos y culturales iniciaron un proceso de aislamiento y protección de esos mismos bienes, que apartó poco a poco los museos de su primera función, dando prioridad a las tareas de investigación, conservación y aumento de las colecciones. El precio que se ha pagado por aquella actitud, inconsciente en muchos casos pero fuertemente elitista, todavía afecta a los museos actuales.

Sin embargo, posiciones críticas hacia el inmovilismo y el aislamiento de los museos surgieron ya desde la revolución de 1968, protagonizadas por grandes pensadores como Adotevi (1972) y Cameron (1970), museólogos como Rivière (1974), Varine (1976) y Desvallées, Bary y Wasserman $(1992,1994)$ que recogieron todos estos trabajos en Vagues, dos libros de referencia para la museología, especialmente en su aspecto de intervención social. De mismo modo, fueron fundamentales iniciativas como la de ICOM en su IX Conferencia Internacional, realizada en Grenoble - Francia, en 1971 y dedicada a los temas: "El museo al servicio del hombre de hoy y del mañana" y "El papel educativo de los museos". Este encuentro fue un espacio abierto de debate y enfrentamiento de ideas entre museólogos innovadores y tradicionalistas. Muchos de los textos presentados por los teóricos de la comunidad mundial de museos, que participaron en aquella reunión, aun hoy sorprenden por su actualidad.

Sorprende por eso que, 50 años después, se hable de la función social del museo como de un pensamiento innovador, sin que parezca estar claro todavía lo que eso significa, y resulta preocupante darse cuenta de que una buena parte de los museos y de sus técnicos y conservadores considera que cumple con su obligación social, abriendo sus puertas y organizando actividades para las escuelas y colegios. Curiosamente, esta propuesta innovadora de dar una función social educativa y de utilidad social a los museos no tuvo gran eco en la museología tradicional, sino que, por el contrario, hemos asistido, en las últimas décadas, a un fenómeno de magnificación del museo como objeto de triunfo político y social, que ha llevado a cometer algunas de las más extrañas paradojas arquitectónicas en la edificación y habilitación de los museos. Nuevos museos, grandes y pequeños, se construyeron olvidando o desconociendo las más elementales reglas organizativas, que implica empezar por el programa museológico y por conocer el encuadramiento humano, social y cultural del territorio envolvente. Reglas que, ya en 1970, G. H. Rivière (1974) presentaba en un documento fundamental denominado "Procedimiento del programa y el proyecto para la construcción de un museo".

El desolador panorama actual de algunos museos, tanto de grandes capitales como de provincias, construidos con frecuencia por motivos electoralistas, y que en algunas ocasiones acapararon presupuestos casi obscenos de construcción, sin calcular los costes posteriores a su apertura, es una peligrosa consecuencia de la no comprensión del museo como un elemento de intervención social y cultural en el medio en que se integra, que debe ser entendido, participado y reconocido como tal por sus utilizadores/clientes.

Irónicamente, la crisis social y económica global, que afecta a nuestras sociedades más desarrolladas, podrá ayudar a resolver algunas de estas contradicciones. De hecho, parece indicar que ya no será posible construir tan fácilmente como antes, por lo menos en la península ibérica, de manera arbitraria y por opción de conveniencia o personalista, instalaciones culturales 
dispersas y costosas, como museos, auditorios y salas de exposiciones, en ciudades o pueblos que apenas pueden sostener y utilizar con regularidad una pequeña parte de lo realizado. Y esto, a pesar de haber enormes agendas de actividades, con poca estrategia y menos público, y que rara vez se discuten previamente con el usuario. También parece que está cerca, por la misma razón, la hora final de las construcciones culturales megalómanas, en las que el coste de mantenimiento del edificio puede ser mayor que la totalidad de sus funciones y sus actividades anuales.

En nuestros países, ha faltado, en los últimos años, capacidad en los profesionales de los museos para abandonar sus posiciones cómodas de conservadores y afirmar la necesidad y la utilidad de la cultura de los museos para los ciudadanos del siglo XXI; ha faltado sensatez en los dirigentes políticos para distribuir con equidad las disponibilidades financieras para la cultura y los museos por todo el territorio y de acuerdo con programas racionales y sustentables en el tiempo; y ha faltado en la academia y en las estructuras educativas la comprensión y la solidaridad necesarias para reforzar con su apoyo el aprendizaje y la defensa de los valores culturales del patrimonio, como un elemento fundamental del conocimiento y de la evolución de las comunidades.

Naturalmente, esta reflexión no cuestiona aquellos cambios positivos que el museo ha realizado, su actitud proactiva para con los visitantes, los esfuerzos de técnicos de museos y profesores para sensibilizar a los alumnos ante la cultura y el patrimonio, o las iniciativas de políticos y gobernantes que han luchado por una gestión saludable y racional de los bienes culturales. Esta reflexión se refiere, sobre todo, al hecho incontrolable de que generaciones de jóvenes continúan transformándose en adultos sin haber adquirido una actitud proactiva ante el patrimonio y los museos. El esfuerzo y los métodos utilizados no parecen suficientemente eficaces para producir resultados visibles y no existe una política metódica de contacto entre el sistema educativo y las organizaciones museológicas y culturales que promueva y permita a los jóvenes adquirir esa actitud. Y mientras no exista una cantidad suficiente de público adulto, interesado y cliente regular de los museos, que justifique a los ojos de tutelas y gobernantes los gastos que un museo genera, los museólogos, los museos, y el patrimonio y la cultura, especialmente en las regiones más alejadas de los grandes centros urbanos, tendrán siempre su actividad en riesgo a cada vuelta política del destino o en cada crisis económica. Y conviene repetir que si esto es preocupante en las grandes ciudades, se vuelve un riesgo de extinción en la intervención municipal y local sobre el patrimonio.

Por eso, y a pesar de los esfuerzos de innovación y actualización de los museos y de sus servicios educativos, para la mayoría de la población, el museo, salvo raras excepciones, o todavía es un lugar serio y aburrido, que se ha visitado una vez, en el mejor de los casos durante la época escolar, y que muy pocas veces ha conseguido generar en estos jóvenes, durante su visita, los deseos de volver. Será conveniente, por tanto, que pongamos los dedos en nuestras heridas e intentemos saber por dónde salir del marasmo cultural en que la cultura y los museos se encuentran, con frecuencia a merced de opciones políticas y conveniencias históricas, como si cultura, educación y patrimonio fuesen asuntos de poner y quitar en cantidad y calidad, en función de la disponibilidad financiera del momento, cuando todos sabemos del retroceso y de las desastrosas consecuencias que esta volubilidad trae para el conjunto social.

Será oportuno también empezar por los problemas de afirmación y defensa de nuestra propia actividad, porque parece que a los museos y museólogos les faltan algunos elementos esenciales para su supervivencia:

- definición clara y reconocida, interna y externamente, de la misión del museo y su función social en el mundo;

- consolidación profesional de sus técnicos y especialistas;

- espacios y medios eficaces de divulgación y promoción de su misión y su actividad;

- integración metódica del aprendizaje del valor del patrimonio y de los museos en el medio educativo;

- capacidad de afirmación junto a las estructuras de poder y de las tutelas de que dependen;

- medios de evaluación que permitan probar su verdadero valor como contribución al desarrollo;

- apoyo regular de las instituciones universitarias para permitir analizar, evaluar y mejorar su papel de intervención en una sociedad en permanente mudanza;

- reconocimiento, por parte de la población, de su utilidad como recurso de crecimiento social y cultural. 
Para poder cambiar este estado de cosas, muchas de las cuales son deficiencias de tipo interno y de responsabilidad de los propios profesionales de los museos, será necesario, tal vez en primer lugar, alterar y mejorar nuestro propio comportamiento como grupo profesional y, además, envolver en el proceso museal a los grupos y personas que influyen en su actividad y funcionamiento, porque, como en todas las organizaciones, sólo la acción concertada y la coexistencia de todos estos grupos y de sus elementos, permitirá a los museos funcionar de forma sostenible. Especialmente, porque parece, como ya apuntamos anteriormente, que los museos no han conseguido todavía probar cabalmente su valor y su utilidad institucional junto a los poderes y las sociedades que los sustentan.

La sostenibilidad de los procesos y la garantía de continuidad en una organización se consiguen cuando existe un grupo humano bien formado $\mathrm{y}$ coherente, con nociones claras sobre su misión y sobre lo que significa el liderazgo, el sentido de la responsabilidad social o la capacidad para tomar decisiones en equipo. Y si esto es importante en cualquier organización de producción, en el caso específico de las organizaciones de servicios, como los museos, la participación de todas las personas que influyen, interna o externamente, en su actividad y su existencia es un componente esencial para su supervivencia.

Los principales grupos de personas que podemos encuadrar en nuestro análisis, además de los curadores, técnicos y empleados que los gestionan y garantizan su funcionamiento son, como ya hemos visto, los poderes políticos que supervisan y deciden sobre la cultura y los museos, los académicos y especialistas universitarios que reflexionan y teorizan sobre ellos y los visitantes y usuarios, incluyendo aquellos que viven alrededor del museo, sus vecinos, y que comparten con ellos territorio y patrimonio.

Hugues de Varine (1976) ya defendía, en los años 70 del siglo pasado, este principio de entendimiento político entre los distintos actores, como él los llamaba, a los elementos y grupos que influencian el museo, considerando este entendimiento como la base esencial de su existencia. Por tanto, si los museólogos quieren ver su actividad y su profesión reconocida y sus iniciativas respetadas, cabe a estos actuar:

- promoviendo la fuerza asociativa que permita crear una orden de museólogos con poder para intervenir en las decisiones sobre el patrimonio y los museos y sobre la carrera profesional de sus técnicos y especialistas;

- consiguiendo, a través de una legislación adecuada, el reconocimiento de los museos y la capacidad de decisión de sus profesionales por parte de los poderes políticos, que con frecuencia intervienen en función de sus propias convicciones, raramente consultando a los profesionales del área. Solo así se comprende que los cargos dirigentes de muchas instituciones culturales y museales sean consideradas "de confianza" o que cualquier político, con o sin conocimiento, pueda decidir el presente o futuro de cualquier museo;

- consiguiendo de las propias tutelas una evaluación cualitativa de la actividad museal, que permita un análisis de desempeño y de resultados más completo que apenas la evaluación cuantitativa del número de visitantes;

- consiguiendo que el mundo educativo admita como elemento integrado de la formación escolar materias dedicadas a la comprensión de la cultura y el patrimonio como elemento fundamental de la construcción del futuro de la sociedad;

- consiguiendo con la comunidad universitaria realizar los programas curriculares que permitan formar profesionales con capacidad técnica y de conocimiento de la realidad social e institucional que les permita ejercer con eficacia su función;

- consiguiendo que las comunidades visitantes y los utilizadores de los museos los reconozcan como interesantes y útiles para su vida, preparando con la colaboración de sus asociaciones y de sus estructuras de representación, las actividades y los modelos de actuación adecuados.

\section{La creación de valor compartido en los museos}

El sistema capitalista está en estado de sitio. En los últimos años, la actividad empresarial se ha visto cada vez más como una de las principales causas de los problemas sociales, ambientales y económicos. Existe una percepción generalizada de que la empresa se nutre a expensas de la comunidad que lo rodea ... El valor compartido ... es la llave que abrirá la próxima ola de innovación y crecimiento de las empresas. Además, se volverá a asociar el éxito de la empresa y el éxito de la co- 
munidad de una manera que fue olvidado en una era en que los enfoques de gestión eran tacaños, de razonamiento inmediatista y de creciente alejamiento entre las instituciones y la sociedad.

\section{Michael E. Porter e Mark R. Kramer (2011)}

Probablemente por motivos que se relacionan con la necesidad de garantizar una rentabilidad imprescindible para el éxito de sus negocios, las organizaciones económicas siempre han conseguido encontrar respuestas a las dificultades de gestión de sus procesos y casi siempre han estado por delante de las grandes decisiones de desarrollo, para bien y para mal, del conjunto social. Naturalmente, estamos hablando de las buenas organizaciones económicas que persiguen algo más que el simple lucro, como es el caso de las que se encaran en el importante artículo que encabeza esta sección del presente texto. Y será bueno que en las áreas de humanidades dejemos de anatematizar a las organizaciones económicas, porque corremos el riesgo de quedarnos separados de la mitad de nuestro mundo en movimiento y marginados en una dependencia financiera poco saludable. De la misma forma que no deberemos olvidar que las organizaciones las hacen las personas y, con las mismas leyes y normas éticas de comportamiento, existen buenas y malas empresas, buenos y malos gobiernos, buenas y malas universidades y buenos y malos museos.

El concepto de creación de valor compartido y de responsabilidad social está exigiendo a las empresas actuales que dejen de ser meras estructuras con fines de lucro y elijan formas de compartir que contribuyan al desarrollo general de la sociedad. La creación de valor significa que ahora no es sólo necesario producir bienes, servicios o productos que generan una respuesta adecuada a las expectativas depositadas previamente por los socios de un negocio, sino también que hay que garantizar el respeto al medio ambiente, prevenir los residuos y generar beneficios para la sociedad en su conjunto.

La creación de valor compartido en las actividades de una organización está así estrechamente vinculada no sólo con las necesidades y la satisfacción de sus clientes, promotores y empleados, sino también con las comunidades que las rodean y con la sociedad en general, a través del reconocimiento por todos de los beneficios que produce. $\mathrm{Y}$ estos beneficios, en términos de conveniencia, precio y calidad de los productos o servicios que ofrece, tienen que ser percibidos bajo un punto de vista racional, económico y emocional por todas las partes involucradas, para que la creación de valor se haga efectiva y produzca los resultados esperados.

Pero, a pesar de que la creación de valor es esencial para el éxito de un negocio a medio y largo plazo, en las organizaciones de producción o servicios de carácter lucrativo, no siempre existe, como se ha probado en las recientes crisis mundiales. Del mismo modo, Porter y Kramer (2011) denuncian la conciencia de la responsabilidad social y de la necesidad de compartir éxitos y fracasos entre los distintos miembros de esas estructuras que, formando parte del mismo juego de una forma complementaria y trabajando juntos, obtendrán resultados satisfactorios y sostenibles para todos y cada uno de sus elementos. Transponiendo estos principios a la museología y considerando que los museos son organizaciones sin fines de lucro, la creación de valor compartido podrá ser un mecanismo de funcionamiento y mejoría continuos que permita, por un lado, garantizar mejores resultados socioculturales y económicos al menor costo posible $\mathrm{y}$, en segundo lugar, producir y demostrar un mayor retorno del esfuerzo realizado, al tiempo que se logra una mayor satisfacción y reconocimiento de todos los elementos que participan en los procesos museales: técnicos, especialistas, políticos y comunidad.

Pero, para que esto suceda, el museo debería, al igual que hacen las organizaciones económicas:

\footnotetext{
- modificar sus modelos y procesos;

- modernizar sus métodos de trabajo;

- diseñar y utilizar modelos de evaluación para mejorar y extender las buenas prácticas;

- buscar el éxito a través de la participación de todos los interesados de forma concertada.
}

\section{Sociomuseología y museos para el siglo XXI}

La Sociomuseología tiene su base en un movimiento nacido en la Universidade Lusófona de Humanidades e Tecnologías de Lisboa, que, desde el inicio de los años 90 del siglo pasado, se dedica a investigar y promover las teorías, experiencias y valores de la museología social y del MINOM/ICOM - Movimiento Internacional 
para una Nueva Museologia. El concepto "Sociomuseología" fue creado por Fernando Santos Neves, antiguo rector de esta universidad y su Centro de Estudios de Sociomuseologia publica regularmente una revista Cadernos de Sociomuseologia ${ }^{l}$, que ya ha editado su número 50 . La Universidad Lusófona promueve también un mestrado y un doctorado en la misma área. En 2012, la Asociación Española de Museólogos publicó su número 53 sobre el mismo tema, recogiendo intervenciones de especialistas y museólogos de distintos ámbitos mundiales. La Sociomuseología pretende recoger y abrazar todos los conceptos y prácticas de este tipo de museología y quiere hacerlos evolucionar y adaptarse a los cambios que las dos últimas décadas de globalización han introducido en todos los ámbitos sociales y culturales, y que han afectado naturalmente a los museos.

Uno de los valores que promueve la Sociomuseologia para el siglo XXI es la mediación cultural, que coloca al museo como un facilitador entre la cultura y la población, promoviendo la participación activa de las comunidades a través del patrimonio y procurando desarrollar hábitos de inclusión, interculturalidad y solidaridad interracial, ante las dificultades y conflictos causados por las constantes migraciones de los pueblos. Este concepto, utilizado por Varine (2011) para las nuevas misiones de los museos en el siglo XXI, incluye también la búsqueda del punto de encuentro entre la democratización cultural y la democracia cultural. La permanente disponibilidad para el cambio, la inclusión como principio democrático, la interdisciplinaridad como modelo de acción y la innovación en la gestión museológica, a través de las tecnologías de Gestión de la Calidad y de herramientas como la CAF Marco Común de Evaluación, son algunos de los principios que mueven a la Sociomuseología en el desempeño de sus programas de investigación y trabajo de campo.

\section{El Observatorio Museo/Escuela: una experiencia de creación de valor compartido en Portugal}

El Observatorio Museo/Escuela ha sido creado en el ámbito del Núcleo de Educación de MINOM - Portugal para acompañar, estudiar y evaluar la relación entre los museos y las escuelas y su misión principal es encontrar y promover mecanismos para garantizar que los jóvenes ganen afecto por los museos y sus valores culturales en los primeros años de su vida escolar, de modo que en la edad adulta conozcan y defiendan el patrimonio y usen la cultura como herramienta de desarrollo social. En este momento, está en curso y en observación una experiencia de creación de valor compartido, que cuenta con la colaboración del Centro de Estudios de Sociomuseologia de la Universidad Lusófona y los ayuntamientos, los museos y las escuelas de tres municipalidades portuguesas de pequeña dimensión.

El observatorio gestiona y analiza sobre todo, con el apoyo de la universidad, los sistemas de evaluación cuantitativa y cualitativa utilizados, como una manera de verificar su eficacia, corregir errores detectados, provocar mejorías al menor costo posible y extender las buenas prácticas. El Observatorio está formado por un grupo de trabajo integrado por conocedores y operacionales del tema a desarrollar y con capacidad de decidir. En este sentido, son miembros del observatorio:

- Tres sociomuseólogos con experiencia en gestión y promoción de museos locales.

- Un responsable municipal del área de educación a nivel de concejalía;

- Un responsable escolar a nivel de dirección;

- Un técnico superior de cada museo participante y un profesor de cada escuela, perteneciente a las clases que se integran en el programa en análisis.

\section{El programa "Héroes del museo por un año"}

Este programa tuvo su origen en las actividades y experiencias de dos museos: el trabajo de evaluación y mejoría continua realizado desde 2009 en el MCCB - Museu da Comunidade Concelhia de Batalha $^{2}$ y el programa de colaboración "Mano a mano", que se inició en 2006 en el Museu do Trajo de São Brás de Alportel $^{3}$, con las escuelas de su zona.

En 2009 y antes de inaugurar el museo en 2011, el MCCB llevó a cabo más de 600 encuestas entre los niños de la localidad (552 encuestas contestadas). El análisis de los resultados consolidaron la opinión ya conocida: serio y antiguo fueron las palabras más utilizadas en la descripción de un museo y reforzaron la necesidad de colaboración con la escuela para promover el cambio. Por su parte, "Mano a mano" pretendía 
optimizar los recursos limitados del museo y utilizarlos de acuerdo con las necesidades educativas de primer ciclo de la educación básica en las escuelas del Municipio de São Brás, promoviendo un esfuerzo por crear hábitos cívicos y culturales en el grupo de edad de 6 a 10 años, como una inversión para el futuro.

El resultados de estas iniciativas y la necesidad de resolver las dificultades surgidas llevaron a la realización piloto de una experiencia en tres museos locales, fuertemente implicados con sus comunidades: la Casa-Museu João Soares de Cortes - bajo la tutela de la Fundación Mário Soares ${ }^{4}$, el Museo del Traje de São Brás - bajo la tutela de la Santa Casa de Misericordia (asociación de asistencia social, con grandes tradiciones en Portugal), y el Museo de Batalla - tutelada por su ayuntamiento.

Entre las muchas razones que aconsejan la participación de los jóvenes estudiantes en el museo, destacamos:

- El escaso conocimiento por parte de los niños, maestros y adultos, del papel que los museos, especialmente los locales, pueden desempeñar en el crecimiento individual y social;

- La imposibilidad de obtener cobertura financiera para garantizar que todos los niños en edad escolar puedan acceder a este conocimiento, sin un programa concreto, continuo, sistemático y de costo accesible;

- La necesidad de abordar las sucesivas crisis, especialmente las actuales, sin perder la capacidad de intervención y la calidad de los servicios prestados.

Dadas estas razones principales, el desarrollo de este programa persigue:

- Procurar la presencia protagonista y continua de los niños en edad escolar por un período de tiempo suficiente para adquirir el conocimiento y afecto por el museo;

- Garantizar, a través de este modelo de intervención, la presencia, la comprensión y el apoyo de los padres y las familias de los niños en el museo;

- Promover entre los jóvenes el valor de la memoria, el patrimonio y la cultura como vehículos de la interculturalidad, la solidaridad y el desarrollo sostenible;
- Garantizar, dentro de un período razonable de tiempo, que todos los jóvenes de una región/área en particular tienen un período suficiente de contacto relacional con el museo para poder considerarlo como propio.

El programa de colaboración tiene las características siguientes:

- Promoción de un programa anual de colaboración entre el museo y una clase concreta de la escuela o colegio (en Portugal la tercera clase del ciclo básico, con niños entre los 10 y 11 años), repitiendo el programa con las mismas clases y distintos niños todos los años;

- Promoción de, por lo menos, una actividad mensual durante todo el año escolar ( 8-9 sesiones combinadas con los profesores previamente y relacionados con los programas escolares en curso), en el museo o en el centro escolar, estando el transporte garantizado desde el principio por el ayuntamiento correspondiente;

- La designación formal del programa "Héroes del Museo por un año " (escogido por los niños del primer programa) pretende garantizar a través de diversas señales externas (distintivo personal del año en curso, diploma, entrada libre incluida su familia, fiesta de apertura y clausura) la participación sistemática de estos niños en las actividades y animación gestión de sus museos.

El desarrollo del programa, que tiene un plano de trabajo anual y una revisión global cada cuatro años, es acompañado y apoyado por el Observatorio y pasa por las siguientes fases:

- Encuesta a niños y adultos de la localidad (a repetir cada 4 anos)

- Análisis acompañado de los resultados

- Realización del programa

- Re-análisis y planificación de mejorías

- Retoma del proceso.

Las características específicas en número y formato de los diversos programas deben ser desarrollados por el museo en colaboración con los profesores de las respectivas escuelas y en función del tipo de museo. Una página dedicada a este tema está disponible para niños y adultos del 
programa, en el sitio MINOM - Portugal, ${ }^{5}$ ya que el propósito principal es permitir compartir experiencias, extender la iniciativa y crear nuevos elementos interesados en la actividad. El objetivo final de este proyecto de colaboración entre elementos y grupos de la sociedad para crear valor compartido en el museo y darle un nuevo protagonismo es un trabajo a medio y largo plazo. Cada año, la misma iniciativa llevada a cabo con un nuevo grupo de niños de la misma clase, puede llegar a tener un retorno eficaz y asequible para la actividad del museo, porque la concentración en un número concreto de participantes y de iniciativas y su naturaleza repetitiva les permite mejorar.

Al mismo tiempo, se está garantizando que todos los niños pasen de forma activa y prolongada por el museo y que estemos ayudando así a crear una ciudadanía más crítica y activa, con un mejor conocimiento del patrimonio y de la cultura, asegurando al mismo tiempo que estos valores se extiendan a toda la comunidad a través de padres y familiares.

Finalmente, puede considerarse como una última meta provocar la expansión del modelo y la adhesión de más museos y escuelas, de manera que se consiga cubrir todo el espacio social de existencia de ambos, en cualquier ámbito territorial. En el Observatorio pensamos que esta puede ser una buena manera de mostrar y probar el valor de un museo, al integrarse en la sociedad, compartiendo esfuerzos y resultados y contribuyendo activamente al desarrollo integral de todos.

\section{Notas}

1. Para consultar on-line los Cadernos de Sociomuseologia usar el [URL: http://revistas.ulusofona.pt/index. $\mathrm{php} /$ cadernosociomuseologia].

2. [URL: http://www.museubatalha.com/].

3. [URL: http://www.google.pt/\#q=museu+do+trajo + de $+\mathrm{s} \% \mathrm{C} 3 \% \mathrm{~A} 3 \mathrm{o}+\mathrm{br} \% \mathrm{C} 3 \% \mathrm{~A} 1 \mathrm{~s}+\mathrm{de}+$ alportel].

4. [URL:http://www.fmsoares.pt/casa_museu/].

5. [URL:http://www.minom-portugal.org/educ/minomeduc.html].

\section{REFERENCIAS Bibliográficas}

Adotevi, A. (1972): Le musée dans les sys 'temes éducatifs et culturels contemporains. Le musée au service des hommes aujourd'hui et demain. Actes de la 9ième Conférence Generale de l'ICOM (ICOM, ed.), Grenoble 1971:19-30.

Cameron, D. (1970): Museums and the world of today. Museum reform in the 1950s and 1960s. ICOM News, 23 (2): 41-45.

Desvallées, A.; De Bary, O.; Wasserman, F. (dir.) (1992, 1994): Vagues Une anthologie de la nouvelle muséologie Vol. 1 et 2. Ed. MNES, Collection Muséologie. Savigny-le-Temple. MNES.

Porter, M.; Kramer, M. (2011): Criação de valor compartilhado. Harvard Business Review, 22: 33. [RLU: http://www.hbrbr.com.br/materia/criacao-de-valor-compartilhado]. Acceso el 13/03/2014.

Rivière, G.H. (1974): Processus du programme et du projet pour la construction d'un musée. En Desvallées, Bary, Wasserman 1992: 317-320.

VARINE, H. (1976): Le musée moderne, conditions e problèmes d'une rénovation. Revista MUSEUM International XXVIII, $n^{\circ}$ 3. Paris: UNESCO: 131-144. [URL: http://unesdoc.unesco.org/ulis/cgi-bin/ExtractPDF.pl?catno=22282\&look=default\&1l=1]. Acceso el 17/3/2014.

VARINE, H. (2011): El museo mediador. Revista de Museología, no 49, Madrid: 16-23. 